\title{
Extraction of Revolving Channels of Drifters around Mesoscale Eddy Centers Based on Spatiotemporal Trajectory Clustering
}

\author{
CHUNYONG MA \\ College of Information Science and Engineering, Ocean University of China, and Laboratory for Regional \\ Oceanography and Numerical Modeling, Qingdao National Laboratory for Marine Science and Technology, \\ Qingdao, China \\ SIQING LI, YANG YANG, AND JIE YANG \\ College of Information Science and Engineering, Ocean University of China, Qingdao, China \\ GE CHEN \\ College of Information Science and Engineering, Ocean University of China, and Laboratory for Regional \\ Oceanography and Numerical Modeling, Qingdao National Laboratory for Marine Science and Technology, \\ Qingdao, China
}

(Manuscript received 27 January 2019, in final form 24 July 2019)

\begin{abstract}
The global oceanic transports of energy, plankton, and other tracers by mesoscale eddies can be estimated by combining satellite altimetry and in situ data. However, the revolving channels of particles entrained by mesoscale eddies, which could help explain the dynamic process of eddies entraining materials, are still unknown. In this study, satellite altimeter and drifter data from 1993 to 2016 are adopted, and the normalized trajectory clustering algorithm (N-TRACLUS) is proposed to extract the revolving channels of drifters. First, the trajectories of drifters are normalized and clustered by using the densitybased spatial clustering of applications with noise (DBSCAN) algorithm. Next, the revolving channels of drifters around the eddy center are extracted. The ring or arc pattern in the middle of a normalized eddy appears when drifters are uninterruptedly entrained by eddies for more than 30 days. Moreover, the revolving channels of drifters in cyclonic eddies are relatively closer to the eddy center than those in anticyclonic eddies. These revolving channels suggest the principal mode of materials' continuous motion processes that are inside eddies.
\end{abstract}

\section{Introduction}

The oceanic transports of heat and materials by mesoscale eddies play an important role in the change of marine environments and regulation of Earth's climate (Farneti et al. 2010; Zhang et al. 2014). In recent years, with the development of marine observation techniques, such as satellite altimeters, moorings, and Argo floats, global distribution of mesoscale eddies has been observed (Chelton et al. 2007, 2011), and scientists have made preliminary estimates of oceanic mass transports by them (Chen et al. 2010; Xu et al. 2014; Dong et al. 2014). However, fewer studies have focused on the dynamic motion of materials inside an eddy. Although

Corresponding author: Ge Chen, gechen@ouc.edu.cn
Zhang et al. (2014) have used closed potential vorticity (PV) contours to identify the trajectory of fluid particles in eddies and indicated that particles would perform a rotating motion around the eddy center, it is still unclear whether the particles' trajectories have any other patterns in a normalized eddy. This study proposed the concept of revolving channels, which can vividly describe the aggregation of "dynamic movement trajectories" of materials in a normalized eddy rather than the "static positions" of materials. Therefore, these revolving channels can be considered as the principal mode of particle's motion inside the eddy.

To extract the revolving channels of particles inside eddies, a large number of tracers and eddies detection data are needed. The distribution of marine chlorophyll and the biomass of zooplankton can reveal 
the characteristics of materials entrained by eddies, and studies show that both of them are aggregated at the edge of eddies (Chelton et al. 2011; Dong et al. 2016; Samuelsen et al. 2012). However, these studies have not focused on trajectory dynamic process of materials entrained by eddies. As a result, they do not directly reveal the dynamic characteristics of materials' motion around the eddy centers. Drifters from the Global Drifter Program (GDP) can reflect the spatiotemporal characteristics of ocean flow fields' motion (Lumpkin 2016; Dong et al. 2014), and the revolving process of drifters tracking around the eddy center can reveal the process of eddies' internal transport. Therefore, drifters are used in this study to extract the revolving channels of particles inside eddies. In addition, satellite altimeter sea level anomaly (SLA) data [provided by the Archiving, Validation, and Interpretation of Satellite Oceanographic Data (AVISO)] are used to detect global eddies. Finally, we use global eddies and drifter trajectories intersection dataset from 1993 to 2016 to extract the revolving channels.

Lagrangian coherent structures (LCS) theories are usually applied to reveal transport dynamic processes and barriers based on continuous flow field data (Haller 2015). LCS has been widely used to reveal the process of ocean mixing, marine transport (d'Ovidio et al. 2004; Beron-Vera et al. 2008, 2010; Rypina et al. 2010), and pollutant dispersion (Lekien et al. 2005; Coulliette et al. 2007; Mezić et al. 2010; Wei et al. 2013). However, the normalized revolving channels cannot be extracted with LCS theory because the flow field calculated by drifters cannot be guaranteed to be continuous after normalization, which is the prerequisite for extracting LCS (Haller and Beron-Vera 2013). Therefore, a clustering algorithm named normalized trajectory clustering algorithm (N-TRACLUS) is proposed to extract the "revolving channels" in this study. In the N-TRACLUS algorithm, trajectories of drifters are normalized and then divided into different clusters. As a result, revolving channels can be extracted and they will clearly show the distribution of drifters' rotating motion trajectories inside eddies.

\section{Data and method}

\section{a. Eddy identification dataset}

In this paper, global satellite altimeter SLA data from 1993 to 2016 provided by the AVISO center are adopted. The SLA data is an up-to-date and delayed-time daily product, with a $1 / 4^{\circ} \times 1 / 4^{\circ}$ spatial resolution. This paper uses the algorithm improved by Liu et al. (2016)
TABLE 1. Number of positions of drifters entrained by eddies.

\begin{tabular}{ccc}
\hline \multicolumn{1}{c}{ Dataset } & $\begin{array}{c}\text { Anticyclonic } \\
\text { eddies }\end{array}$ & $\begin{array}{c}\text { Cyclonic } \\
\text { eddies }\end{array}$ \\
\hline $\begin{array}{c}\text { Number of positions of drifters } \\
\text { tracking in eddy for less than }\end{array}$ & 2303027 & 2455755 \\
$\quad \begin{array}{l}\text { 30 days } \\
\text { Number of positions of drifters } \\
\text { tracking in eddy for 30-60 days }\end{array}$ & 410265 & 623393 \\
$\begin{array}{l}\text { Number of positions of drifters } \\
\text { tracking in eddy for more than }\end{array}$ & 120551 & 202265 \\
$\quad$ 60 days & & \\
\hline
\end{tabular}

for identification of mesoscale eddies from global satellite altimetry data. In this algorithm, SLA contours are calculated with parallel computing technology, and eddy boundaries corresponding to the maximum ground rotation speed can be extracted (Liu et al. 2016).

\section{b. The dataset that contains the intersections of eddies and drifters}

The drifter data in this study were from the GDP of the National Oceanic and Atmospheric Administration (NOAA), with a temporal resolution of $6 \mathrm{~h}$. Quality control is performed on the data, which includes the identification number of drifters, date, time, longitude, latitude, eastward velocity, northward velocity, speed, and sea temperature.

The data from 1993 to 2016 of global mesoscale eddies and drifters are adopted to obtain a new dataset that contains the intersections of eddies and drifters. Only the data between $66^{\circ} \mathrm{N}$ and $66^{\circ} \mathrm{S}$ are used. Based on the statistics of the intersection dataset, the time during which drifters rotate for a cycle around the eddy centers is about 30 days. The dataset was divided into three parts according to the time that drifters continuously tracking in eddies: less than 30 days, 30 to 60 days, and more than 60 days. The corresponding total numbers of drifters' positions are shown in Table 1, and the global distributions are shown in Fig. 1.

In this paper, we use these intersection data to track eddies. It is assumed that there are two continuous intersection data with a same drifter. Eddies in these two intersection data are named E1 and E2. The distance between their center is marked as center - distance, and their polarities are Type1 and Type2, respectively. If their parameters satisfy the conditions of Eqs. (1) and (2), then E1 and E2 are supposed to be the same eddy:

$$
\begin{aligned}
& \text { center }- \text { distance } \leq 0.5^{\circ}, \\
& \text { Type } 1=\text { Type } 2 .
\end{aligned}
$$

In the above eddy tracking process, it is uncertain that a drifter is tracking the same eddy throughout the 

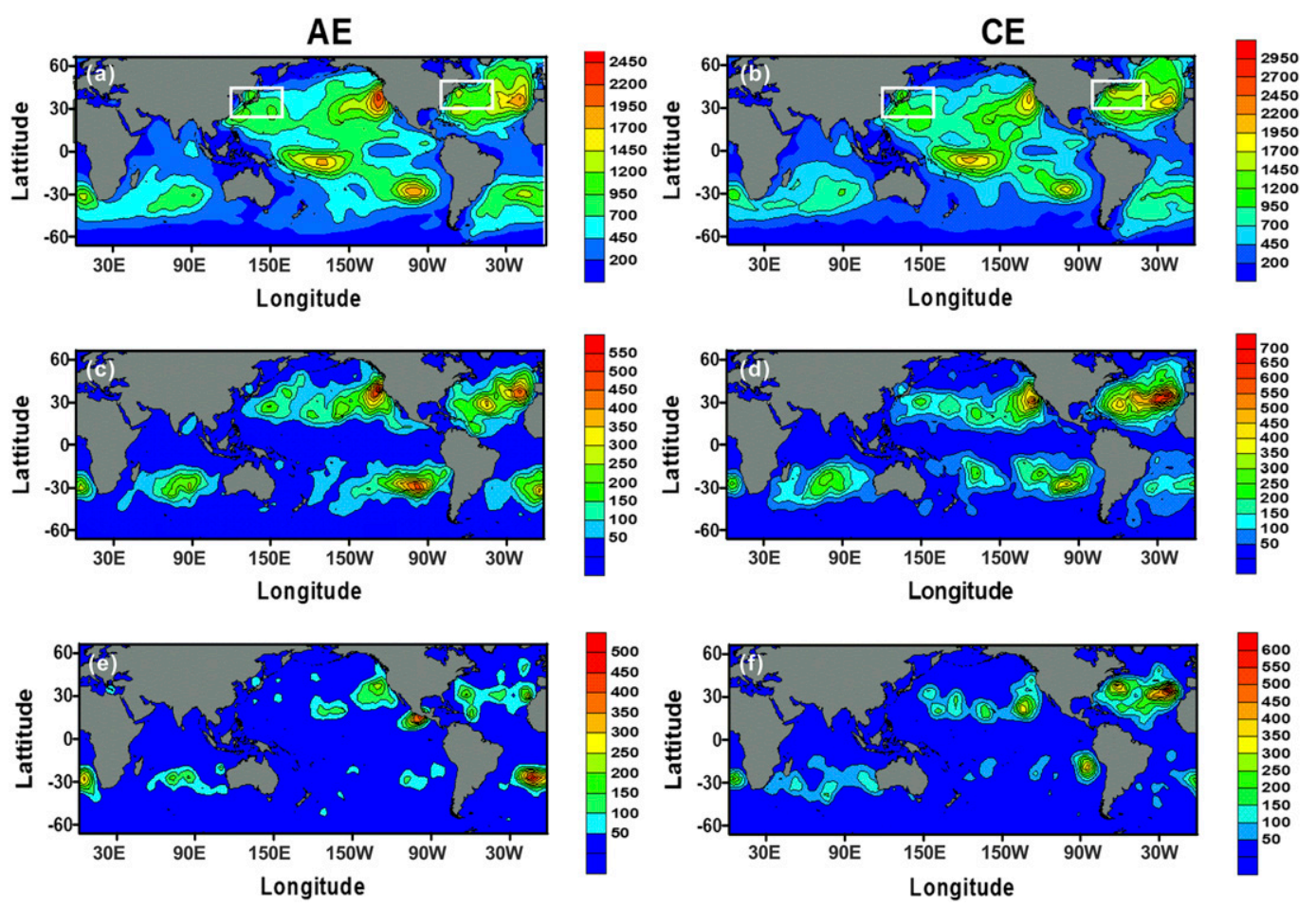

FIG. 1. Global distribution of drifters that are entrained by eddies. (a),(b) The global distribution of drifters that are entrained by anticyclonic and cyclonic eddies for less than 30 days, respectively. The white rectangular areas show the two experimental areas in section 4. (c),(d) The global distribution of drifters that are entrained by anticyclonic and cyclonic eddies for 30-60 days, respectively. (e),(f) The global distribution of drifters that are entrained by anticyclonic and cyclonic eddies for more than 60 days, respectively.

time. We verified this eddy tracking algorithm with a eddy tracking method using satellite altimeter data (Sun et al. 2017). It is found that $5 \%$ of the eddies tracking results are different. However, the spatiotemporal sampling of drifters is continuous, and our judgement conditions are strict enough, while satellite altimeter products may be inaccurate or miss eddies due to their sparse spatiotemporal sampling (Amores et al. 2018). The tracking data of eddies obtained by our algorithm are therefore considered to be ground truth for calculation and analysis in this paper.

\section{c. The method of extracting revolving channels of drifters}

The dynamic trajectories of drifters are clustered by the N-TRACLUS algorithm, which is based on the density-based spatial clustering of applications with noise (DBSCAN) algorithm. The DBSCAN algorithm is a typical spatial clustering algorithm based on density. The principle of the DBSCAN algorithm is to calculate the density distribution for each point in space, gather the points of similar density, and put them in the same cluster. In contrast to the $K$-means algorithm, it does not require the predetermination of the number of clusters, and shape of clusters can be maintained. The DBSCAN algorithm can effectively remove noise points and find arbitrary shapes of spatial clusters (Ester et al. 1996). The N-TRACLUS algorithm adopted in this study was optimized based on the DBSCAN algorithm. The trajectories of drifters are normalized and divided into line segments according to the minimum description length (MDL) principle. Next, these line segments are clustered based on the algorithm of DBSCAN. The N-TRACLUS algorithm is described below.

\section{1) NORMALIZING THE TRAJECTORIES OF DRIFTERS}

The trajectories of drifters in different mesoscale eddies have a substantially different scale for the azimuth angle and the distance between drifter's positions and the eddy centers. Therefore, the trajectories of drifters must be normalized before clustering. The relative positions among drifters, the eddy center and the boundaries of eddy are used to normalize the trajectories of the drifters entrained by mesoscale eddies. First, the intersected point of the eddy's boundary and straight line that connects the eddy center to the drifter's position is calculated. Next, the distance from the eddy 
center to the intersected point is obtained as the standard, which can be used to normalize the distance and azimuth angle between the drifter's position and the eddy center. The normalized radial distance $x$ can be obtained by Eq. (3), and the normalized zonal distance $y$ can be obtained by Eq. (4):

$$
\begin{aligned}
& x=\frac{\text { Dist } 1}{\text { Dist2 }} \times \sin \alpha, \\
& y=\frac{\text { Dist1 }}{\text { Dist2 }} \times \cos \alpha .
\end{aligned}
$$

Here, Dist1 represents the distance between the eddy center and drifter's position, while Dist2 represents the length of a straight line from the eddy center to the eddy's boundary via the drifter position (Fig. 2). The $\alpha$ represents the azimuth angle between the eddy center and the drifters. The schematic diagrams of the normalization of drifters' trajectories are shown in Fig. 3 (Fig. 3a shows the original trajectories of the drifters, while Fig. $3 \mathrm{~b}$ shows the normalized trajectories).

\section{2) DIVIDING THE TRAJECTORIES OF DRIFTERS INTO SEGMENTS}

The MDL principle is used in this study to divide the trajectories of drifters entrained by eddies into line segments. The MDL principle consists of two components, L1 and L2. The L1 represents the length of the hypothesis description (in bits), and L2 represents the length of the data description when encoded with the help of the hypothesis (in bits). In our trajectory partitioning problem, a hypothesis corresponds to a set of trajectory partitions (Lee et al. 2007).

Suppose the trajectory (TL) of a drifter in an eddy consists of a set of line segments, $p^{1}, p^{2}, p^{3}, \ldots, p^{n}$, then L1 can be obtained by Eq. (5). Here, len $\left(p^{j}, p^{(j+1)}\right)$ represents the Euclidean distance between the segments $p^{j}$ and $p^{(j+1)}$. We formulate L2 by Eq. (6), which represents the summation of the difference between the trajectory TL and a set of its trajectory partitions:

$$
\begin{aligned}
& \mathrm{L} 1=\sum_{j=1}^{n-1} \log _{2}\left[\operatorname{len}\left(p^{j}, p^{(j+1)}\right)\right], \\
& \mathrm{L} 2=\sum_{j=1}^{n} \operatorname{sum}_{j !=k}^{n}\left\{\log _{2}\left[d_{v}\left(p^{j}, p^{k}\right)\right]+\log _{2}\left[d_{\theta}\left(p^{j}, p^{k}\right)\right]\right\} .
\end{aligned}
$$

To measure the difference, we use the sum of the perpendicular distance and the angular distance. We do not consider the parallel distance since a trajectory encloses its trajectory partitions.

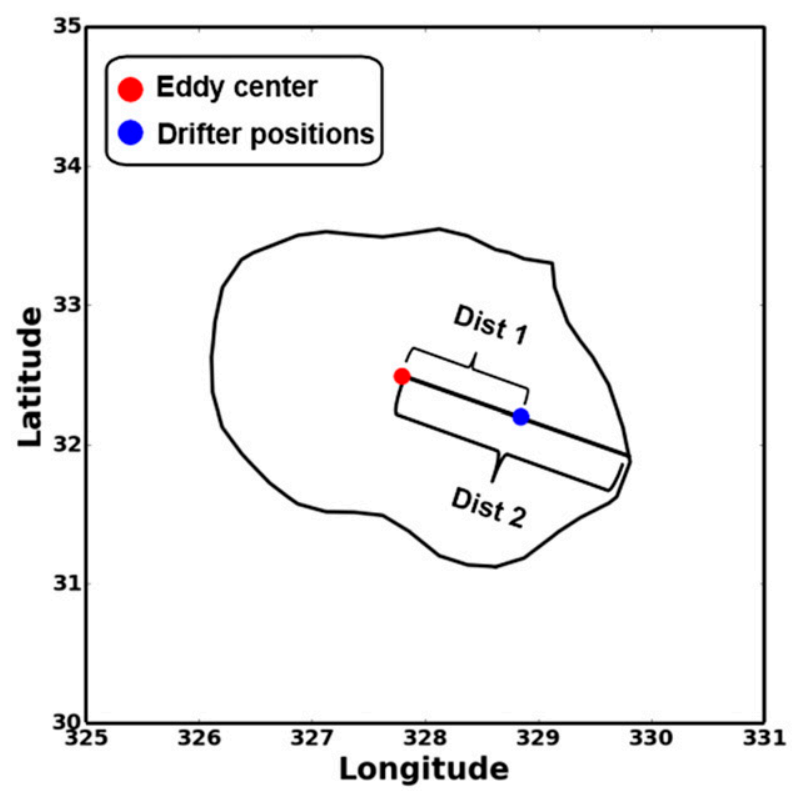

FIG. 2. The schematic diagram of parameters Dist1 and Dist2 during the progress of normalizing drifters' trajectories. Dist1 represents the distance from the eddy center to drifter's position. Dist 2 represents the distance from the eddy center to the boundary.

\section{3) Clustering The Line Segments}

To clustering the line segments, two parameters need to be given first: 1) Eps, which represents the search radius of each line segment; and 2) Minpts, which represents the threshold of the number of accessible line segments in the neighborhood with radius Eps (Du et al. 2016; Lee et al. 2007). In this study, the Eps value is 0.12, which is the mean normalized distance of drifters moving daily. The Minpts value is 8 for the trajectory clustering of drifters entrained by eddies for less than 30 days, 14 for 30 to 60 days, and 20 for more than 60 days. The values of these two parameters are shown in Table 2. For each unclassified segment $p$, the Euclidean distance between it and other segments in the neighborhood, with radius Eps, is calculated to determine whether segment $p$ is the primary line segment, whose number of accessible line segments in the neighborhood with radius Eps is greater than the given threshold parameter Minpts. In this study, the distance between two line segments $p^{i}$ and $p^{j}$ is defined by Eq. (7). Here, $d_{v}\left(p^{i}, p^{j}\right), d_{p}\left(p^{i}, p^{j}\right)$, and $d_{\theta}\left(p^{i}, p^{j}\right)$ represent the perpendicular distance, parallel distance, and angle distance, respectively (as shown in Fig. 4). The methods of calculating the above three kinds of distance are given by Eqs. (8)-(10), where $w_{v}, w_{p}$, and $w_{\theta}$ represent the weights of the perpendicular distance, parallel distance, and angular distance, respectively: 

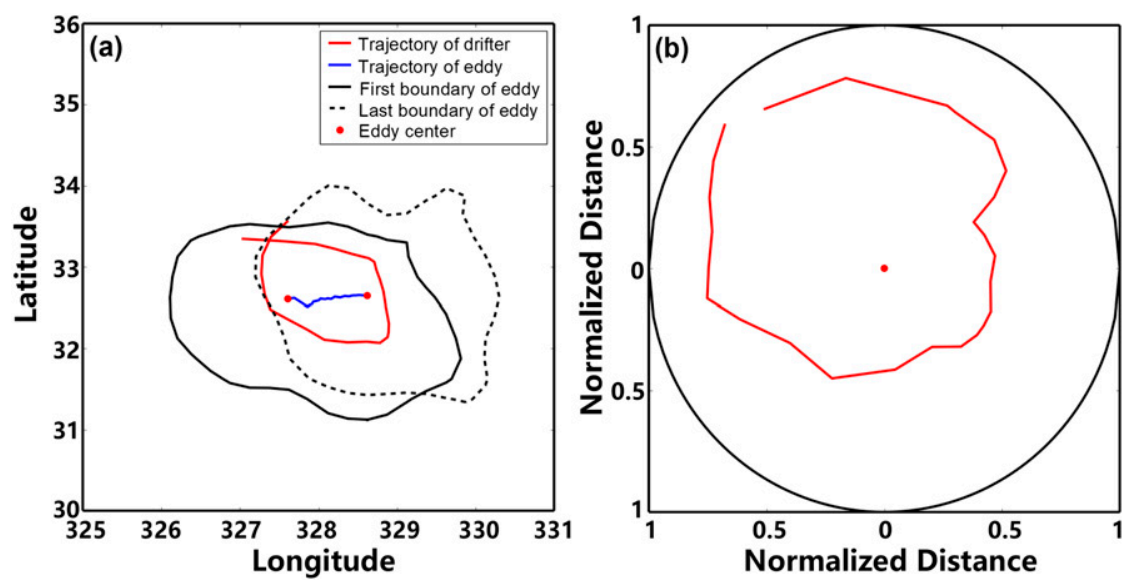

FIG. 3. The schematic diagram of the normalization of drifters' trajectories. (a) The original trajectory of a drifter. The solid black curve represents the boundary of eddy when the drifter enters the eddy, and the dashed black curve represents the boundary of eddy when the drifter leaves the eddy. The solid red curve represents the trajectory of the drifter, and the solid blue curve represents the trajectory of eddy center. (b) The normalized trajectory of the drifter. The solid black curve represents the boundary of the normalized eddy, and the solid red curve represents the normalized trajectory of the drifter. The red dots represent the eddy center in both (a) and (b).

$$
\begin{aligned}
\mathrm{DT}\left(p^{i}, p^{j}\right)= & w_{v} \times d_{v}\left(p^{i}, p^{j}\right)+w_{p} \times d_{p}\left(p^{i}, p^{j}\right) \\
& +w_{\theta} \times d_{\theta}\left(p^{i}, p^{j}\right), \\
d_{v}\left(p^{i}, p^{j}\right)= & \left(P_{v 1}^{2}+P_{v 2}^{2}\right) /\left(P_{v 1}+P_{v 2}\right), \\
d_{p}\left(p^{i}, p^{j}\right)= & \operatorname{MIN}\left(P_{p 1}, P_{p 2}\right), \\
d_{\theta}\left(p^{i}, p^{j}\right)= & |P|_{2} \sin \theta .
\end{aligned}
$$

After calculating the distance and statistics, if $p$ represents the primary line segment, the following steps are performed: find all line segments where $p$ can enter the neighborhood with radius Eps and add these line segments to the cluster. If the newly added line segments are not classified, then add them to a new cluster for further extension. If $p$ is not the primary line segment, it will not be added to the cluster. Finally, the total number of line segments in each cluster is calculated, and if its value is less than the threshold Minpts, the cluster is discarded.

\section{4) EXTRACTING THE REVOLVING CHANNELS OF DRIFTERS}

Existing studies have used the trajectory partitionand-group method to cluster the trajectories of mesoscale eddies, in which perpendicular lines to the line segments of each cluster are taken to obtain the representative trajectories (Lee et al. 2007). However, this method only supports straight motion, so it cannot be used to extract the revolving channels of drifters rotating around the eddy centers. We extend this algorithm to support circular motion and it is optimized as follows: the rays are taken from the eddy center to the boundary as auxiliary line, with a resolution of $1^{\circ}$. These rays intersect the line segments of each cluster, and the number of intersected points of rays and line segments are calculated. If the number is larger than Minpts, the mean value of the intersected point of this cluster is calculated as the final points of the clustering result. Then the revolving channels can be obtained by connecting the final points. However, if the number is less than the threshold Minpts, this cluster will be discarded.

\section{Result}

\section{a. Statistical result of eddies in the dataset}

The statistics of eddy kinetic energy (EKE) and radius of mesoscale eddies in the dataset are first analyzed, and

TABLE 2. List of parameter values of N-TRACLUS clustering algorithm used in this paper.

\begin{tabular}{ccc}
\hline Dataset & Eps & Minpts \\
\hline $\begin{array}{c}\text { Value of drifters tracking in eddy } \\
\text { for less than 30 days }\end{array}$ & 0.12 & 8 \\
$\begin{array}{l}\text { Value of drifters tracking in eddy } \\
\text { for 30-60 days }\end{array}$ & 0.12 & 14 \\
$\begin{array}{l}\text { Value of drifters tracking in eddy } \\
\text { for more than 60 days }\end{array}$ & 0.12 & 20 \\
\hline
\end{tabular}




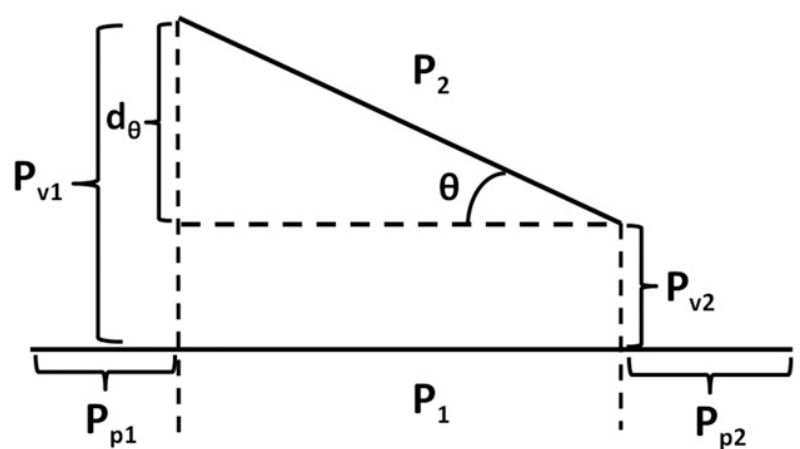

FIG. 4. Three components of the distance function for line segments: P1 and P2 represent the line segments of drifters' trajectories, $\theta$ represents the angle between $\mathrm{P} 1$ and $\mathrm{P} 2, d_{\theta}$ represents the angle distance between $\mathrm{P} 1$ and $\mathrm{P} 2, P_{v 1}$ and $P_{v 2}$ are used for calculating the perpendicular distance, and $P_{\mathrm{p} 1}$ and $P_{\mathrm{p} 2}$ are used for calculating the parallel distance.

the results are shown in Fig. 5 and Fig. 6. The following can be seen from the figures: 1) When EKEs are less than $100 \mathrm{~cm}^{2} \mathrm{~s}^{-2}$ or radii are less than $100 \mathrm{~km}$, the proportion of eddies that entrain drifters for more than 60 days (red bar) is smaller than other types of eddies (blue and green bars). On the contrary, when EKEs are more than $100 \mathrm{~cm}^{2} \mathrm{~s}^{-2}$ or radii are more than $100 \mathrm{~km}$, eddies that entrain drifters for more than 60 days account for the largest proportion. 2) The maximum proportion of anticyclonic (cyclonic) eddies that entrain drifters for more than 60 days appears when the EKE is in range of 100-200 (0-100) $\mathrm{cm}^{2} \mathrm{~s}^{-2}$ and the radius is in range of 100-150 $(50-100) \mathrm{km}$, respectively. This indicates that anticyclonic eddies may require bigger EKE and radius than cyclonic eddies, when they entrain drifters for a long time.

\section{b. Results of revolving channel extracting}

Figure 7 shows the clustering process of eight sample trajectories of drifters entrained by mesoscale eddies. First, the MDL principle is used to process the original trajectories of drifters (as shown in Fig. 7a), and the line segments are obtained (as shown in Fig. 7b). The MDL principle can use the fewest line segments to accurately reveal the characteristics of the original trajectories of drifters and achieve the unified accuracy and simplicity of the division of trajectories, which can reveal both the instantaneous movement and the general movement of drifters in eddies. Second, based on the two parameters set in the algorithm, Eps and Minpts, the line segments in Fig. $7 \mathrm{~b}$ are filtrated and clustered, and the line segments whose number of accessible line segments in a neighborhood with radius Eps is less than Minpts are removed (the solid blue lines in Fig. 7b). Finally, we used auxiliary line ray as shown by the orange line in Fig. 7c. The rays are taken from the eddy center to the boundary, with a resolution of $1^{\circ}$. After the rays intersect with the line segments, the mean values of the intersected points are taken and sequentially connected to represent the result of the trajectory clustering (the solid red curve in Fig. 7c). The optimized trajectory clustering algorithm N-TRACLUS can detect common motion characteristics from a trajectory dataset. Therefore, it can extract the revolving channels of drifters' circular motion around the eddy centers form drifter's trajectory dataset.

The results of extracting the revolving channels of global drifters are shown in Figs. 8a-f. First, the extracted channels of the drifters entrained by mesoscale eddies for less than 30 days are shown in Figs. 8a and $8 \mathrm{~d}$. Regardless of whether the eddies are anticyclonic or cyclonic, the channels extracted from the trajectory clustering present disorderly characteristics, indicating it is difficult for drifters to perform a complete circular motion within 30 days. Among them, we analyzed the trajectories data that are located on the passage through the eddy centers. To obtain the main factors influencing drifters' direction, the daily averaged grid wind field data (2000-09) provided by Remote Sensing Systems (RSS) and the surface geostrophic velocities
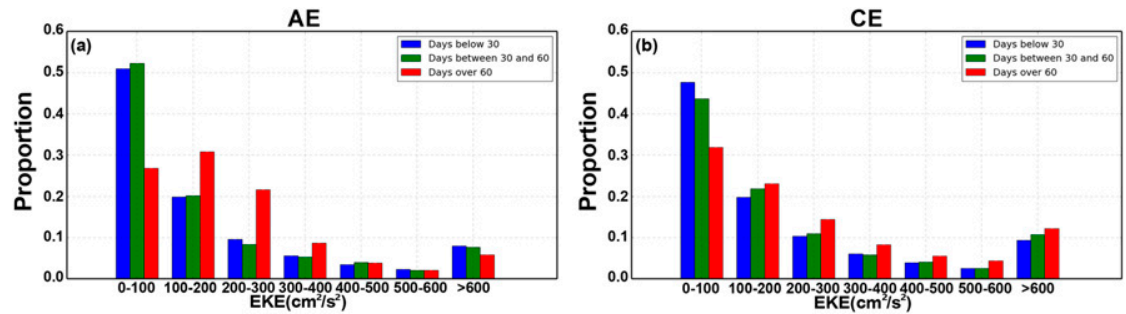

FIG. 5. The bar diagrams of EKE's distribution of eddies that entrain drifters for different duration. (a) The distribution of anticyclonic eddies' EKE, and (b) the distribution of cyclonic eddies' EKE. The blue bars represent the eddies that entrain drifters for less than 30 days. The green bars represent the eddies that entrain drifters for 30-60 days. And the red bars represent the eddies that entrain drifters for more than 60 days. 

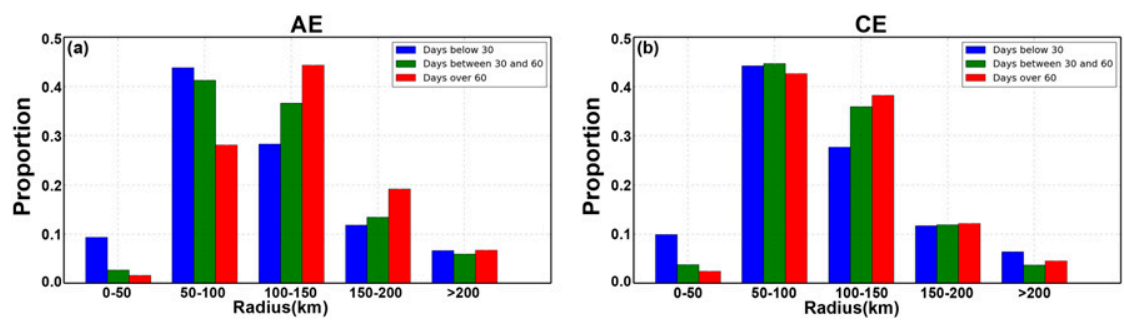

FIG. 6. The bar diagrams of radius' distribution of eddies that entrain drifters for different duration. (a) The distribution of anticyclonic eddies' radius, and (b) the distribution of cyclonic eddies' radius. The blue bars represent the eddies that entrain drifters for less than 30 days. The green bars represent the eddies that entrain drifters for 30-60 days. And the red bars represent the eddies that entrain drifters for more than 60 days.

data provided by AVISO are used. Analysis shows that the average angle $\left(87^{\circ}\right)$ between the direction of the drifters' motion and the wind field is smaller than the average angle $\left(99^{\circ}\right)$ between that of drifters' motion and surface geostrophic current, which means that when drifters have been entrained by mesoscale eddies for a short time (shorter than 30 days), the influence of the wind is slightly greater than that of surface geostrophic currents. Second, revolving channels that extracted from the drifters entrained by mesoscale eddies for more than 30 days and less than 60 days are shown in Figs. $8 \mathrm{~b}$ and $8 \mathrm{e}$. The channels extracted from drifters entrained by eddies for more than 30 days present a ring or arc pattern. There are two revolving channels channel 1 and channel 2 in anticyclonic eddies, which can be seen in Fig. $8 \mathrm{~b}$, and the density distribution of the trajectories that are grouped into these two channels is shown in Fig. 9a. For quantitative description of the extracted revolving channels, two methods of proportions calculation have been designed:
1) Proportion of trajectories. The meaning of a "trajectory" is the overall motion path of a drifter continuously entrained by the same eddy, and the method for calculating the proportion is the ratio of trajectories participating in the clustering to the total number of trajectories. It should be explained that as long as one of the segments of trajectory participates in the clustering process of a certain channel, the flag of whether this trajectory participates in this channel clustering is marked as "true," and one trajectory may be marked as "true" in different channels at the same time, so the sum of the proportion is likely to be greater than $100 \%$.

2) Proportion of positions. In this method, the ratio of drifters' positions that are in the clustering of channels to the total number is calculated as the proportion of positions. Different from the proportion of trajectories, a position of drifter can only be calculated in a channel, so the sum of the proportion is less than or equal to $100 \%$.
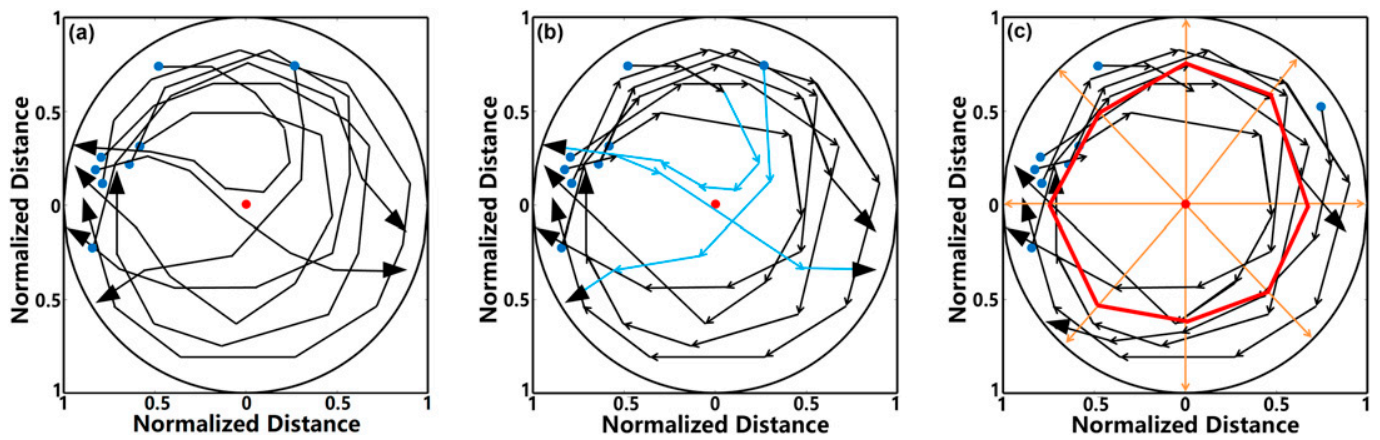

FIG. 7. The schematic diagram of the trajectory clustering algorithm. (a) The normalized trajectories of drifters. (b) The line segments derived from the normalized trajectories with the MDL principle. The solid blue curves represent the line segments that were removed in the clustering. (c) The result of trajectories clustering. The solid black curves represent the normalized trajectories of drifters. The solid orange curves are the rays taken from the eddy center to the boundary as auxiliary line, with a resolution of $1^{\circ}$ [only eight curves are shown in (c)]. These rays intersect the line segments of each cluster, and the mean value of the intersected point of each cluster were calculated as the final points of the clustering result. Then, the revolving channels could be obtained by connecting the final points, which are represented as solid red curves in (c). 

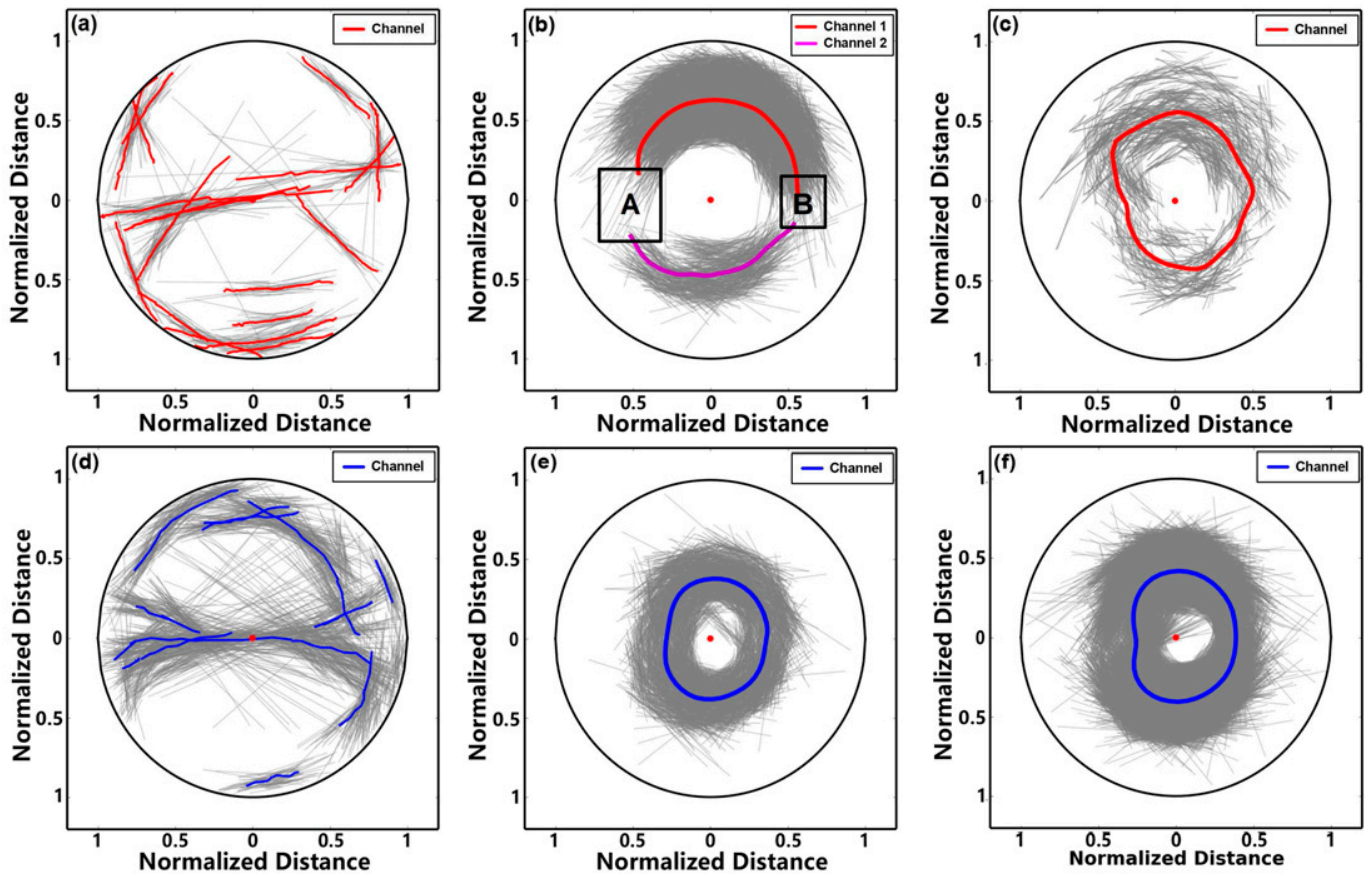

FIG. 8. The revolving channels of drifters in global eddies. (a),(d) The revolving channels of drifters that are entrained by anticyclonic and cyclonic eddies for less than 30 days, respectively. (b),(e) The revolving channels of drifters that are entrained by anticyclonic and cyclonic eddies for 30-60 days, respectively. (c),(f) The revolving channels of drifters that are entrained by anticyclonic and cyclonic eddies for more than 60 days, respectively. The solid red curves and the solid magenta curve represent the revolving channels of drifters in anticyclonic eddies. The solid blue curves represent the revolving channels of drifters in cyclonic eddies. The solid gray lines represent the line segments of trajectories.

For channel 1 and channel 2 in Fig. 8b, the proportions of trajectories are $72.5 \%$ and $62.8 \%$, and the proportions of positions are $33.4 \%$ and $21.1 \%$, respectively, which are shown in Table 3. These two channels can be regarded as fragments of a complete revolving channel, and the two breakpoints are further discussed in section 4. For drifters entrained by cyclonic eddies, a complete revolving channel is extracted from the clustering results, and the density distribution of trajectories participating in this channel is shown in Fig. 9b. For this revolving channel, the proportion of trajectories is $78.4 \%$, and the proportion of positions is $66.7 \%$ (also shown in Table 3 ). These statistical results also show that these revolving channels can be considered as the principal mode of particle's motion inside eddy. Finally, it is found that the normalized distance between the revolving channel and the eddy center in anticyclonic eddies is in the range of 0.55 to 0.63 , while it is in the range of 0.28 to 0.44 in cyclonic eddies. Compared with anticyclonic eddies, the revolving channels of drifters in cyclonic eddies is closer to the center of the eddy.

Figures $8 \mathrm{c}$ and $8 \mathrm{f}$ show the extracted revolving channels from the clustering results of the drifters' trajectories, which are entrained by mesoscale eddies for more than 60 days, and the density distribution of the trajectories participating in the channels is shown in Figs. 9c and 9d. For the extracted revolving channels in anticyclonic and cyclonic eddies, the proportions of trajectories are $75.2 \%$ and $81.6 \%$, and the proportions of positions are $55.8 \%$ and $56.7 \%$, respectively. These proportions of trajectories and positions are shown in Table 4. Similarly, the revolving channel of drifters in cyclonic eddies is closer to the eddy center than that in anticyclonic eddies; the normalized distance between the revolving channel and the eddy center in anticyclonic eddies is in the range of 0.42 to 0.61 , while it is in the range of 0.27 to 0.42 in cyclonic eddies. Meanwhile, the density distribution of involved trajectories in Fig. 9 also shows that trajectories of drifters are more concentrated in the north-south direction of the eddies, while they are sparse in the east-west direction.

The above clustering results show that revolving channels around the eddy center can be extracted from the trajectories of drifters entrained by mesoscale eddies for more than 30 days. In these channels, drifters can be entrained by mesoscale eddies in a circular motion and 

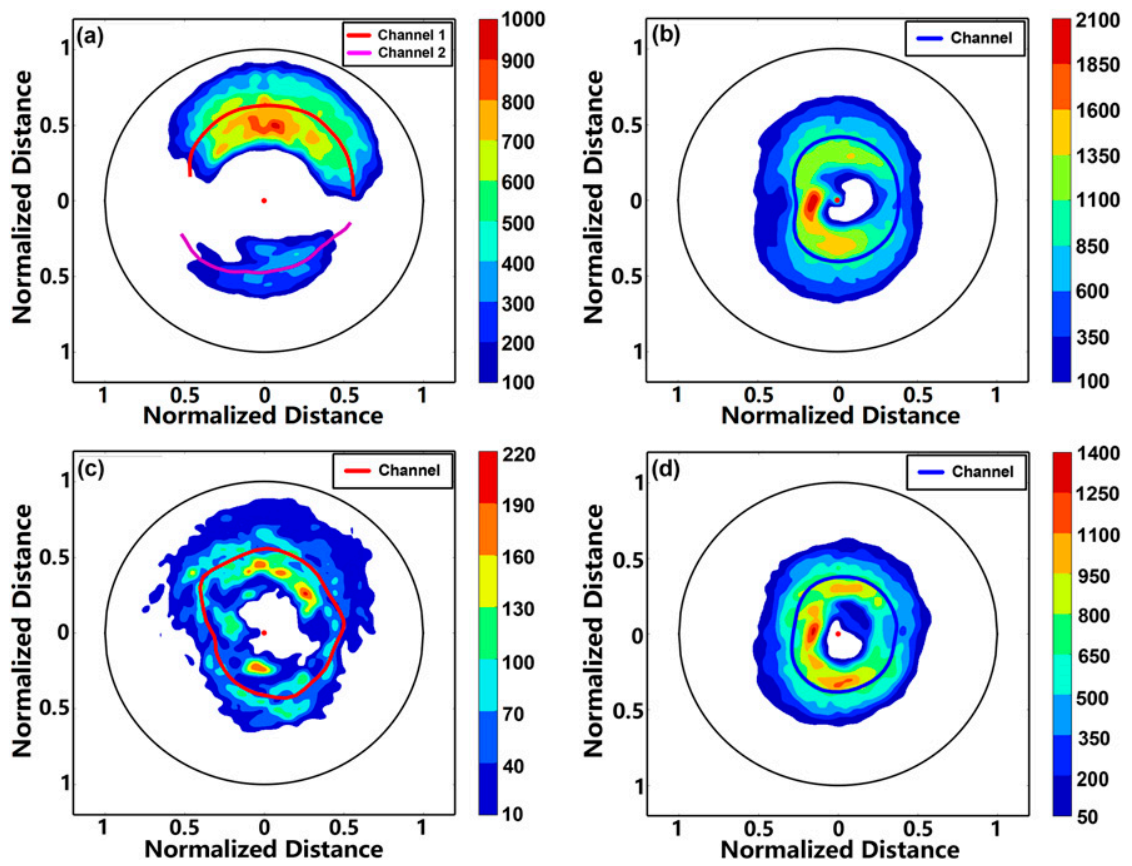

FIG. 9. The density distribution of the trajectories that are grouped into the revolving channels. (a),(b) The trajectories' density distribution of drifters that are entrained by anticyclonic and cyclonic eddies for 30-60 days, respectively, and (c),(d) the trajectories' density distribution of drifters that are entrained by anticyclonic and cyclonic eddies for more than 60 days, respectively. The solid red curves and the solid magenta curve represent the revolving channels of drifters in anticyclonic eddies. The solid blue curves represent the revolving channels of drifters in cyclonic eddies.

they have difficulty leaving the eddies or dramatically changing direction. In addition, the statistics of the drifters entrained by mesoscale eddies for more than 60 days show that there are 142 and 265 trajectories in the anticyclonic and cyclonic eddies, respectively. Therefore, compared with the anticyclonic eddies, the cyclonic eddies can entrain drifters for longer.

\section{Discussion and implications}

Revolving channels represent the principal common features of drifters' trajectories inside eddies. The eddy channels show different patterns according to different eddy polarities and different regions. We will discuss the following three aspects of patterns and their implications:

(i) The ring or arc patterns appear in the middle of normalized eddy center and edge, as shown in Fig. 8.
Existing research indicates that zooplankton and chlorophyll have an aggregation effect at the edges of eddies (Samuelsen et al. 2012; Dong et al. 2016; Wang et al. 2018). Unlike the pattern that particles aggregate near the eddy edge, the channels of drifters entrained by eddies for more than 30 days are approximately midway between the eddy center and the edge. These channels can reflect eddies' radial momentum, which is mainly related to vorticity and Rossby number of eddies (Zhang et al. 2015). It is suggested that the balance position of particles' radial motion in upper-ocean eddies should be midway between the eddy center and the edge. Xu et al. (2016) have put 17 Argo floats in the northwest Pacific region, and realized the tracking observation of typical mesoscale eddies. Argo float (2901566) with the longest tracking time has tracked eddies for more than 170 days,

TABLE 3. For revolving channels of drifters that are entrained by eddies for 30-60 days, the proportion of trajectories and positions.

\begin{tabular}{lccc}
\hline \hline \multicolumn{1}{c}{ Proportion } & Channel 1 in anticyclonic eddies & Channel 2 in anticyclonic eddies & Channel in cyclonic eddies \\
\hline Proportion of trajectories & $72.5 \%$ & $62.8 \%$ & $78.4 \%$ \\
Proportion of positions & $33.4 \%$ & $21.1 \%$ & $66.7 \%$ \\
\hline
\end{tabular}


TABLE 4. For revolving channels of drifters that are entrained by eddies for more than 60 days, the proportion of trajectories and positions.

\begin{tabular}{lcc}
\hline \hline \multicolumn{1}{c}{ Proportion } & $\begin{array}{c}\text { Channel in } \\
\text { anticyclonic eddies }\end{array}$ & $\begin{array}{c}\text { Channel in } \\
\text { cyclonic eddies }\end{array}$ \\
\hline Proportion of trajectories & $75.2 \%$ & $81.6 \%$ \\
Proportion of positions & $55.8 \%$ & $56.7 \%$ \\
\hline
\end{tabular}

and its parking depth was $1000 \mathrm{~m}$. Its average normalized distance from the eddy edge to the Argo is 0.53 (Xu et al. 2016). Therefore, it can be seen that the radial motion balance position of the material in subsurface eddies may also be located midway between the eddy center and the eddy edge.

(ii) Compared with anticyclonic eddies, particles' normalized trajectories in cyclonic eddies have a more continuous pattern. As shown in Fig. 8b, there are two breakpoints in anticyclonic revolving channels extracted by trajectory clustering. Therefore, the normalized trajectory data is used to analyze the motion state in the area of two breakpoints $\mathrm{A}$ and $\mathrm{B}$. The results show that drifters tend to move toward the center or boundary of the mesoscale eddies in the area of breakpoints $\mathrm{A}$ and $\mathrm{B}$ in Fig. 8b, and the probability of moving toward the center of the eddies is higher. For example, in Figs. 10a-c, drifters tend to move toward the center of the mesoscale eddies at breakpoint A, while drifters tend to shift toward the edge of the mesoscale eddies at breakpoints $A$ and B in Figs. 10d-f, and some drifters even began to break away from the eddies. The dramatic changes in the direction of the drifters' motion make two breakpoints appear in the revolving channels of drifters entrained by anticyclonic eddies for 30 to 60 days. For further analysis, the horizontal structure of normalized mesoscale eddy is divided into eight quadrants (as shown in Fig. 11) in this study. The distribution of the last five positions of a trajectory of drifter entrained by anticyclonic eddies will be discussed as the positions where drifters leave eddies. Statistics are made for positions where drifters leave eddies according to the northern and southern hemispheres, and the results are shown in Figs. 12a and 12b. It can be seen from Figs. 12a and 12b that both in the southern and the Northern Hemisphere, there are the most positions where drifters move away from eddies in the $1 / 4 / 5 / 8$ quadrant, especially in the Northern Hemisphere. Furthermore, these four quadrants also correspond to areas $\mathrm{A}$ and $\mathrm{B}$ where the two breakpoints
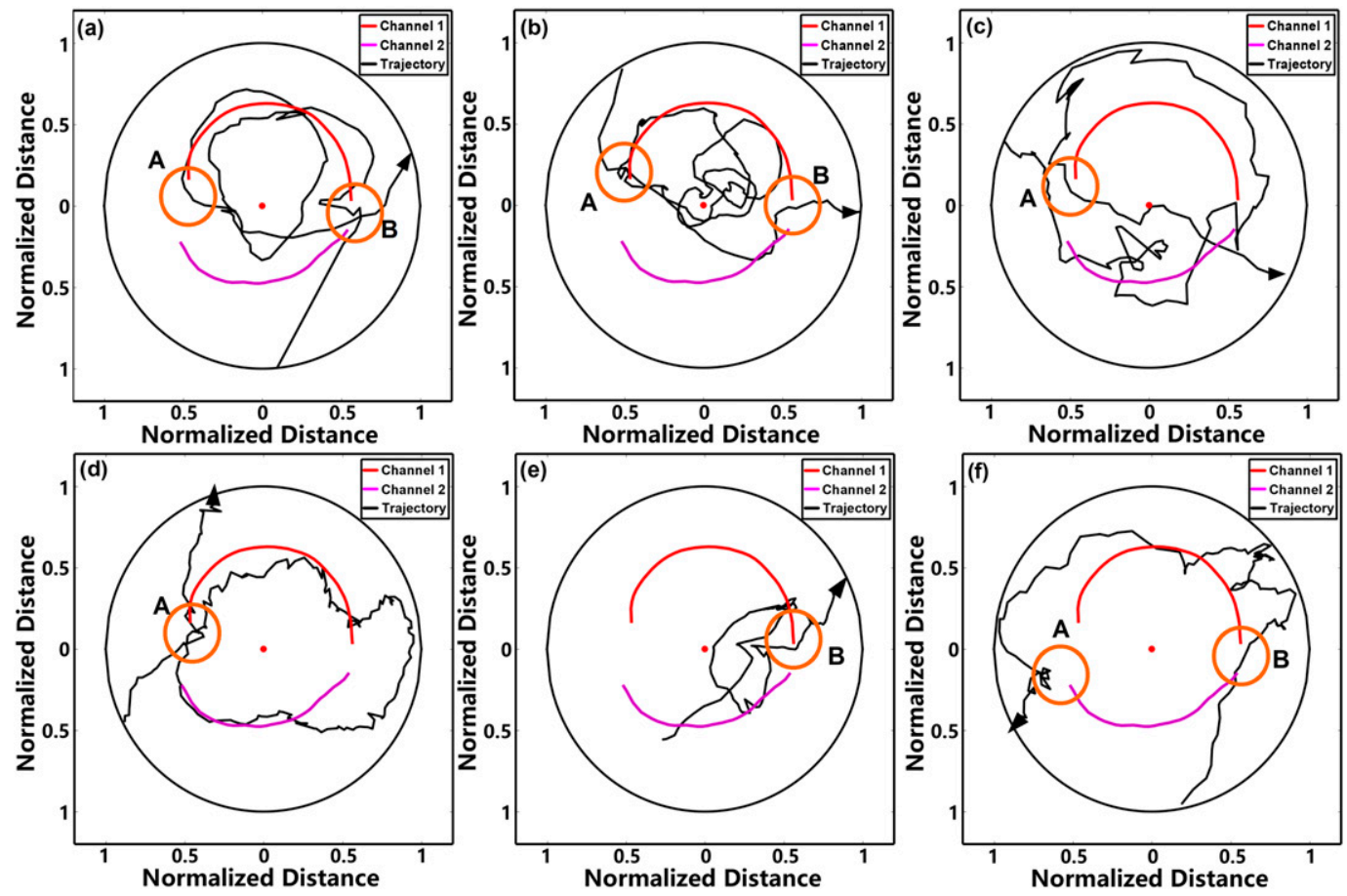

FIG. 10. Examples of the trajectories of drifters entrained by anticyclonic eddies for 30-60 days. The solid red curves and solid magenta curves represent the extracted revolving channels of drifters. The solid black curves represent the trajectories of drifters. 


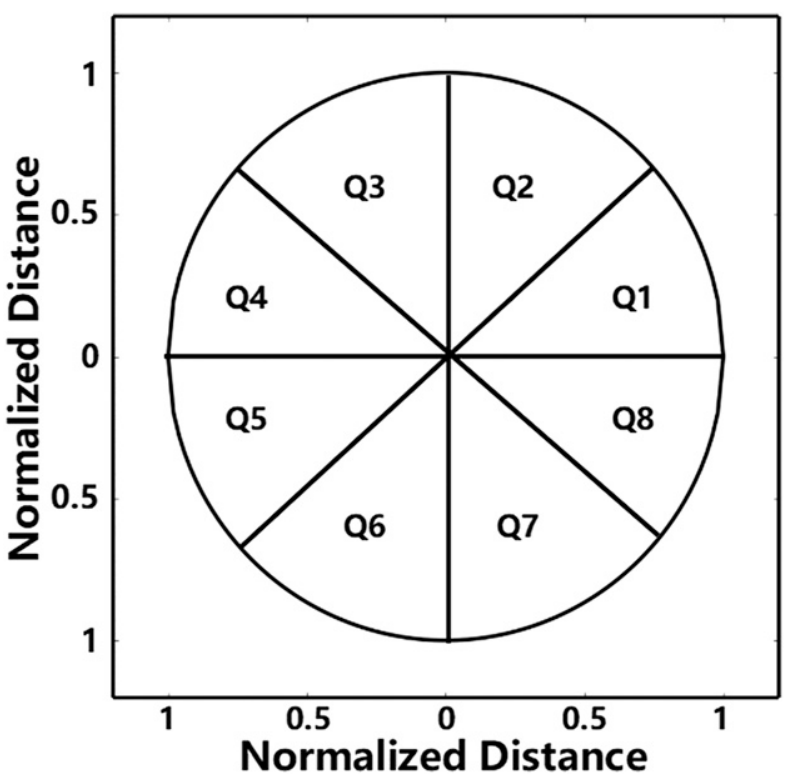

FIG. 11. The schematic diagram of the eight quadrants in a normalized eddy.

are in the revolving channels of drifters entrained by anticyclonic eddies for 30 to 60 days (as shown in Fig. $8 \mathrm{~b}$ ), which provides evidence for the two breakpoints pattern.

The upper-ocean eddies generate mostly by geostrophic motion, as well as ageostrophic motion related to direct wind action in the Ekman layer and to submesoscale dynamics at scales smaller than the Rossby deformation radius. (Chelton et al. 1998; McGillicuddy et al. 2007). Furthermore, eddies may be generated from the shedding of current (Zhang et al. 2016), and turbulence is always included in eddies (Smith et al. 2016). Many aspects of upper-ocean dynamics are still unknown. Previous studies have indicated that the anticyclonic motion of drifters show higher coherences with the wind, through the Ekman response of the upper ocean (Rio and Hernandez 2003; Elipot 2006). In anticyclonic eddies, drifters are more likely to make anticyclonic motion. Therefore, the revolving motion of drifters entrained by anticyclonic eddies are more affected by wind. It may be the main reason of that the channels of anticyclonic eddies are not continuous enough and two breakpoints are formed. In addition, Griffa et al. (2008) calculated global drifters' ring trajectories data showing that drifters' revolving motion around the center in cyclonic eddies has a longer duration and a smaller radius, which is consistent with the results of this study (Griffa et al. 2008). (iii) In addition to extracting the global revolving channels of drifters in eddies using N-TRACLUS algorithm, regional revolving channels also can be extracted based on regional intersection data. To study the difference of patterns between regions, two regions (the northwest Pacific and the North Atlantic), which are shown in white rectangular areas in Fig. 1, are selected for channel extraction experiments. These two regions have in recent years become a focus area for research on mesoscale eddies because of Kuroshio and the Gulf Stream (Andersson et al. 2011; Chen et al. 2010). Therefore, the trajectories of drifters entrained by eddies in the northwest Pacific $\left(10^{\circ}-40^{\circ} \mathrm{N}, 120^{\circ}-\right.$ $\left.150^{\circ} \mathrm{E}\right)$ and the North Atlantic $\left(20^{\circ}-50^{\circ} \mathrm{N}, 70^{\circ}-\right.$ $40^{\circ} \mathrm{W}$ ) were clustered to extract the revolving channels in this study. Here, since the trajectories of drifters in eddies with a time of more than 60 days account for a weak proportion, we selected the trajectories of drifters entrained by eddies in these two regions with a time of more than 30 days for the clustering and extraction of the revolving channels. In the northwest Pacific, there are 135 trajectories of drifters in the anticyclonic eddies and 256 trajectories of drifters in the cyclonic eddies, while in the North Atlantic, there are 278 trajectories of drifters in the anticyclonic eddies and 434 trajectories of drifters in the cyclonic eddies.

The results of the revolving channels' extraction in the northwest Pacific and the North Atlantic are shown in Fig. 13. Compared with the northwest Pacific, the revolving channels of drifters in the North Atlantic are closer to the eddy center. In the North Atlantic, the normalized distances between the channel and eddy center in anticyclonic and cyclonic eddies are in the ranges of 0.43 to 0.52 and 0.28 to 0.42 , respectively, while in the northwest Pacific, these distances are in the ranges of 0.52 to 0.66 and 0.43 to 0.53 , respectively. This experiment can verify the following two patterns, the first one is the ring pattern in the middle of the eddy center and the eddy edge; the second one is that the channel of cyclonic eddies is closer to the eddy center than the channel of the anticyclonic eddies. To find the relationship between revolving channels and ocean dynamics, we calculate the eddies' average Rossby number in these two regions. The Rossby numbers in the northwest Pacific and the North Atlantic are 0.16 and 0.08 , respectively. This suggests that when eddies' Rossby numbers are smaller, the revolving channel is closer to the eddy centers. This result is consistent with particles' 

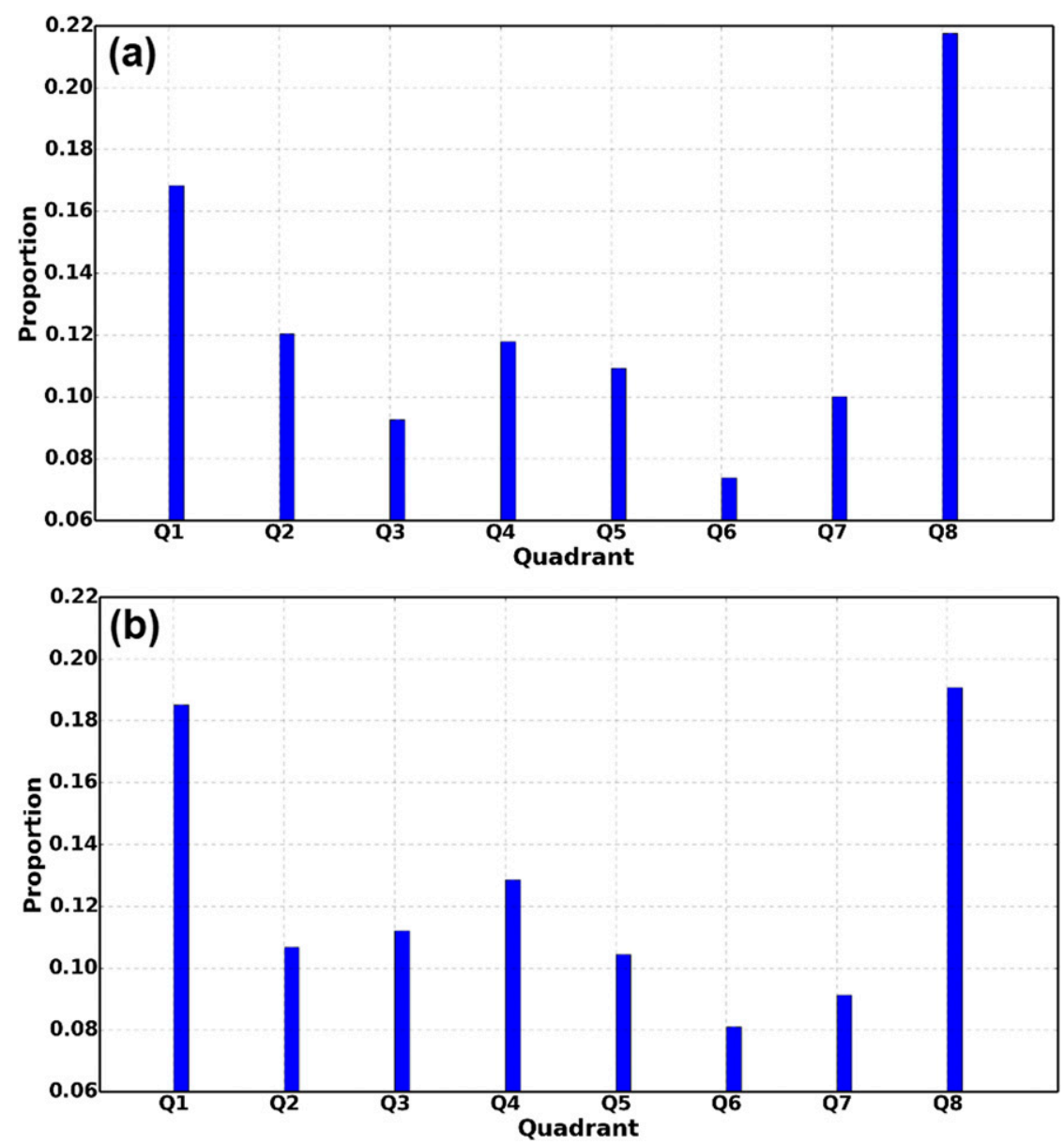

FIG. 12. (a) The proportion of the leaving position of drifters that are entrained by anticyclonic eddies for 30-60 days in each quadrant in the Southern Hemisphere; (b) the proportion of the leaving position of drifters that are entrained by anticyclonic eddies for 30-60 days in each quadrant in the Northern Hemisphere.

dynamical radial displacement theory in the eddy, as described by Zhang et al. (2015).

\section{Conclusions}

Existing studies have fully indicated that mesoscale eddies have strong effect on the oceanic mass transports, which has been preliminarily estimated (Dong et al. 2014; Zhang et al. 2014). This study uses N-TRACLUS algorithm to extract the revolving channels of drifters in anticyclonic and cyclonic eddies. These revolving channels cannot only reveal the spatial distribution of materials entrained by eddies, but also reflect also reflect the dynamic motion process of particles in eddies.

In summary, the study uses composite analysis and spatiotemporal trajectory clustering techniques to extract the principal modes of the drifters' continuous motion in eddies. These principal modes reflect the main characteristics of the transport process inside eddies. The channels extracted from the trajectories of drifters entrained by eddies for less than 30 days are disorderly and unable to reveal the characteristics of circular motion. When drifters are continuously rotating around the eddy center inside an eddy for more than 30 days, the ring or arc pattern is obvious. And the normalized distances of this ring or arc pattern and eddy center is approximately 0.5 , which means that when the materials continuously make a revolving motion around the eddy center, the stable position of the revolving movement in eddies is in the middle of eddy instead of the edge. In addition, it can be clearly seen that the normalized trajectories of the drifters inside cyclonic eddies are more continuous and closer to the eddy center, while the flow field in the anticyclonic eddy is greater affected by the Ekman flow, making it difficult for the normalized trajectory of 

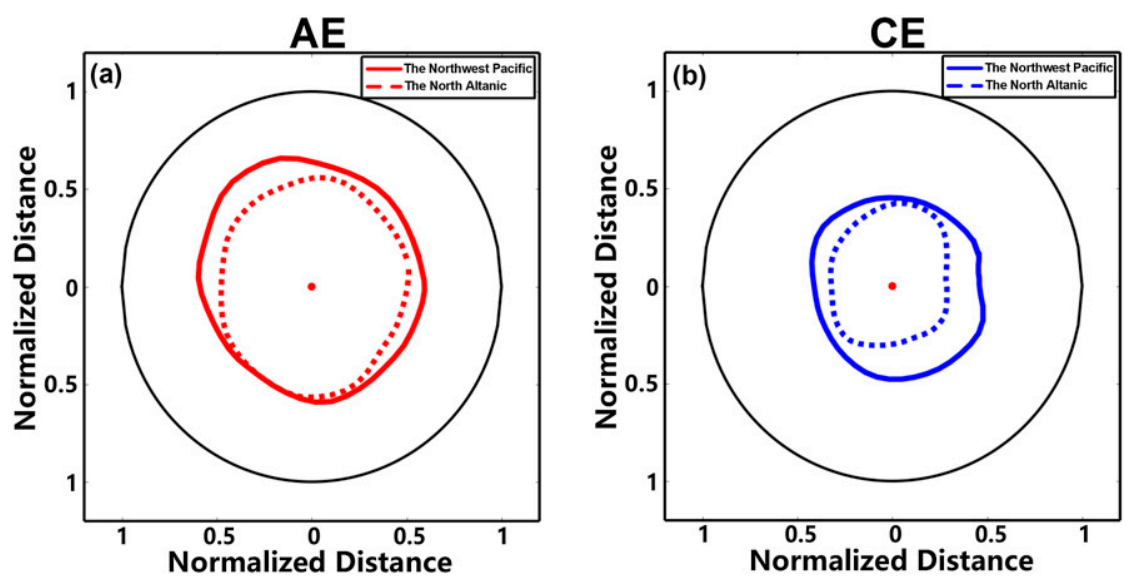

FIG. 13. The extracted revolving channels of drifters that are entrained by eddies in the North Atlantic and the northwest Pacific. (a) The revolving channels of drifters in the anticyclonic eddies. The solid red curve represents the revolving channel in the northwest Pacific, and the dashed red curve represents the revolving channel in the North Atlantic. (b) The revolving channels of drifters in the cyclonic eddies. The solid blue curve represents the revolving channel in the northwest Pacific, and the dashed blue curve represents the revolving channel in the North Atlantic.

drifters in the anticyclonic eddy to maintain a consistent circular mode. The distinction of the channels reflects the difference in the eddy dynamics process, and the connection between the eddy dynamics and the channel characteristics should be further explored in future work.

Acknowledgments. QuikScat and SeaWinds data were produced by Remote Sensing Systems with funding from the NASA Ocean Vector Winds Science Team. The altimeter products were produced by Ssalto/Duacs and distributed by Aviso+. Drifter data were provided by the Global Drifter Program. This research was funded by the Marine S\&T Fund of Shandong Province for Pilot National Laboratory for Marine Science and Technology (Qingdao): 2018SDKJ0102-8; Fundamental Research Funds for the Central Universities: 201762005; National Natural Science Foundation of China: 41906155, 41527901.

\section{REFERENCES}

Amores, A., G. Jordà, T. Arsouze, and J. Le Sommer, 2018: Up to what extent can we characterize ocean eddies using presentday gridded altimetric products? J. Geophys. Res. Oceans, $\mathbf{1 2 3}$, 7220-7236, https://doi.org/10.1029/2018JC014140.

Andersson, M., K. Orvik, J. LaCasce, I. Koszalka, and C. Mauritzen, 2011: Variability of the Norwegian Atlantic Current and associated eddy field from surface drifters. J. Geophys. Res., 116, L15606, https://doi.org/10.1029/ 2011JC007078.

Beron-Vera, F. J., M. J. Olascoaga, and G. J. Goni, 2008: Oceanic mesoscale eddies as revealed by Lagrangian coherent structures. Geophys. Res. Lett., 35, L12603, https://doi.org/10.1029/ 2008 GL033957.
,-- , and — 2010: Surface ocean mixing inferred from different multisatellite altimetry measurements. J. Phys. Oceanogr., 40, 2466-2480, https://doi.org/10.1175/2010JPO4458.1.

Chelton, D. B., R. A. DeSzoeke, M. G. Schlax, K. El Naggar, and N. Siwertz, 1998: Geographical variability of the first baroclinic Rossby radius of deformation. J. Phys. Oceanogr., 28, 433-460, https://doi.org/10.1175/1520-0485(1998)028<0433: GVOTFB $>2.0 . \mathrm{CO} ; 2$.

—, M. G. Schlax, R. M. Samelson, and R. A. de Szoeke, 2007: Global observations of large oceanic eddies. Geophys. Res. Lett., 34, L15606, https://doi.org/10.1029/2007GL030812.

,-- , and _ 2011: Global observations of nonlinear mesoscale eddies. Prog. Oceanogr., 91, 167-216, https://doi.org/ 10.1016/j.pocean.2011.01.002.

Chen, G., Y. Hou, X. Chu, and P. Qi, 2010: Vertical structure and evolution of the Luzon warm eddy. Chin. J. Oceanol. Limnol., 28, 955-961, https://doi.org/10.1007/s00343-010-9040-3.

Coulliette, C., F. Lekien, J. D. Paduan, G. Haller, and J. E. Marsden, 2007: Optimal pollution mitigation in Monterey Bay based on coastal radar data and nonlinear dynamics. Environ. Sci. Technol., 41, 6562-6572, https://doi.org/10.1021/ es0630691.

Dong, C., J. C. McWilliams, Y. Liu, and D. Chen, 2014: Global heat and salt transports by eddy movement. Nat. Commun., 5, 3294, https://doi.org/10.1038/ncomms4294.

__ X. X. Jiang, G. Xu, J. Ji, X. Lin, W. Sun, and S. Wang, 2016: Automated eddy detection using geometric approach, eddy datasets and their application. Adv. Mar. Sci., 12, 371-379.

d'Ovidio, F., V. Fernández, E. Hernández-García, and C. López, 2004: Mixing structures in the Mediterranean Sea from finitesize Lyapunov exponents. Geophys. Res. Lett., 31, L17203, https://doi.org/10.1029/2004GL020328.

Du, Y., D. Wu, F. Liang, J. Yi, Y. Mo, Z. He, and T. Pei, 2016: Major migration corridors of mesoscale ocean eddies in the South China Sea from 1992 to 2012. J. Mar. Syst., 158, 173-181, https://doi.org/10.1016/j.jmarsys.2016.01.013.

Elipot, S., 2006: Spectral characterization of Ekman velocities in the Southern Ocean based on surface drifter trajectories. 
Ph.D. thesis, Scripps Institution of Oceanography, University of California, San Diego, 140 pp.

Ester, M., H.-P. Kriegel, J. Sander, and X. Xu, 1996: A densitybased algorithm for discovering clusters a density-based algorithm for discovering clusters in large spatial databases with noise. Int. Conf. on Knowledge Discovery and Data Mining, Portland, OR, Association for Computing Machinery, 226-231.

Farneti, R., T. L. Delworth, A. J. Rosati, S. M. Griffies, and F. Zeng, 2010: The role of mesoscale eddies in the rectification of the Southern Ocean response to climate change. J. Phys. Oceanogr., 40, 1539-1557, https://doi.org/10.1175/ 2010JPO4353.1.

Griffa, A., R. Lumpkin, and M. Veneziani, 2008: Cyclonic and anticyclonic motion in the upper ocean. Geophys. Res. Lett., 35, L01608, https://doi.org/10.1029/2007GL032100.

Haller, G., 2015: Lagrangian coherent structures. Annu. Rev. Fluid Mech., 47, 137-162, https://doi.org/10.1146/annurevfluid-010313-141322.

, and F. J. Beron-Vera, 2013: Coherent Lagrangian vortices: The black holes of turbulence. J. Fluid Mech., 731, R4, https:// doi.org/10.1017/jfm.2013.391.

Lee, J.-G., J. Han, and K.-Y. Whang, 2007: Trajectory clustering: A partition-and-group framework. Proc. 2007 SIGMOD Int. Conf. on Management of Data, Beijing, China, Association for Computing Machinery, 593-604.

Lekien, F., C. Coulliette, A. J. Mariano, E. H. Ryan, L. K. Shay, G. Haller, and J. Marsden, 2005: Pollution release tied to invariant manifolds: A case study for the coast of Florida. Physica D, 210, 1-20, https://doi.org/10.1016/ j.physd.2005.06.023.

Liu, Y., G. Chen, M. Sun, S. Liu, and F. Tian, 2016: A parallel SLA-based algorithm for global mesoscale eddy identification. J. Atmos. Oceanic Technol., 33, 2743-2754, https://doi.org/ 10.1175/JTECH-D-16-0033.1.

Lumpkin, R., 2016: Global characteristics of coherent vortices from surface drifter trajectories. J. Geophys. Res. Oceans, 121, 1306-1321, https://doi.org/10.1002/2015JC011435.

McGillicuddy, D. J., and Coauthors, 2007: Eddy/wind interactions stimulate extraordinary mid-ocean plankton blooms. Science, 316, 1021-1026, https://doi.org/10.1126/ science. 1136256.

Mezić, I., S. Loire, V. A. Fonoberov, and P. Hogan, 2010: A new mixing diagnostic and Gulf oil spill movement. Science, 330, 486-489, https://doi.org/10.1126/science.1194607.
Rio, M. H., and F. Hernandez, 2003: High-frequency response of wind-driven currents measured by drifting buoys and altimetry over the world ocean. J. Geophys. Res., 108, 3283, https:// doi.org/10.1029/2002JC001655.

Rypina, I. I., L. J. Pratt, J. Pullen, J. Levin, and A. L. Gordon, 2010: Chaotic advection in an archipelago. J. Phys. Oceanogr., 40, 1988-2006, https://doi.org/10.1175/2010JPO4336.1.

Samuelsen, A., S. S. Hjøllo, J. A. Johannessen, and R. Patel, 2012: Particle aggregation at the edges of anticyclonic eddies and implications for distribution of biomass. Ocean Sci., 8, 389400, https://doi.org/10.5194/os-8-389-2012.

Smith, K. M., P. E. Hamlington, and B. Fox-Kemper, 2016: Effects of submesoscale turbulence on ocean tracers. J. Geophys. Res. Oceans, 121, 908-933, https://doi.org/ 10.1002/2015JC011089.

Sun, M., F. Tian, Y. Liu, and G. Chen, 2017: An improved automatic algorithm for global eddy tracking using satellite altimeter data. Remote Sens., 9, 206, https://doi.org/10.3390/ rs9030206.

Wang, Y., H.-R. Zhang, F. Chai, and Y. Yuan, 2018: Impact of mesoscale eddies on chlorophyll variability off the coast of Chile. PLOS ONE, 13, e0203598, https://doi.org/10.1371/ journal.pone.0203598.

Wei, X., P. Ni, and H. Zhan, 2013: Monitoring cooling water discharge using Lagrangian coherent structures: A case study in Daya Bay, China. Mar. Pollut. Bull., 75, 105-113, https:// doi.org/10.1016/j.marpolbul.2013.07.056.

Xu, C., X.-D. Shang, and R. X. Huang, 2014: Horizontal eddy energy flux in the world oceans diagnosed from altimetry data. Sci. Rep., 4, 5316, https://doi.org/10.1038/srep05316.

Xu, L., P. Li, S.-P. Xie, Q. Liu, C. Liu, and W. Gao, 2016: Observing mesoscale eddy effects on mode-water subduction and transport in the North Pacific. Nat. Commun., 7, 10505, https:// doi.org/10.1038/ncomms10505.

Zhang, W.-Z., H. Xue, F. Chai, and Q. Ni, 2015: Dynamical processes within an anticyclonic eddy revealed from Argo floats. Geophys. Res. Lett., 42, 2342-2350, https://doi.org/10.1002/ 2015 GL063120.

Zhang, Z., J. Tian, B. Qiu, W. Zhao, P. Chang, D. Wu, and X. Wan, 2016: Observed 3D structure, generation, and dissipation of oceanic mesoscale eddies in the South China Sea. Sci. Rep., 6, 24349, https://doi.org/10.1038/srep24349.

Zhang, Z., W. Wang, and B. Qiu, 2014: Oceanic mass transport by mesoscale eddies. Science, 345, 322-324, https://doi.org/10.1126/ science. 1252418 . 